\title{
Metachronous occurrence of collagenous colitis and ulcerative colitis
}

\author{
F M Giardiello, F W Jackson, A J Lazenby
}

\begin{abstract}
Collagenous colitis and ulcerative colitis are distinct disorders. A 67 year old woman with clinical and histological evidence of collagenous colitis had an abrupt symptomatic exacerbation while taking anti-inflammatory treatment with sulphasalazine and prednisone. Repeat colorectal endoscopy showed active mucosal inflammation and colonic biopsy specimens were consistent with active ulcerative colitis. After bowel rest, total parenteral nutrition, intensification of the anti-inflammatory regimen, and withdrawal of nonsteroidal anti-inflammatory drugs (which she had taken continuously for osteoarthritis) diarrhoea abated. Colorectal biopsy specimens obtained when the patient's symptoms had improved showed inactive ulcerative colitis with no evidence of collagenous colitis. This may be the first case to be reported of the metachronous association of collagenous and ulcerative colitis.
\end{abstract}

We report on a patient with typical clinical and histopathological features of collagenous colitis whose disease abruptly evolved into active ulcerative colitis. After medical treatment colorectal biopsy specimens showed inactive ulcerative colitis. This is the first case, to our knowledge, of the metachronous association of collagenous and ulcerative colitis.

\section{Case report}

A 67 year old woman was admitted to hospital in July 1989 because she was passing 10 12 watery, non-bloody stools a day. Her past medical history included degenerative joint disease of the knees, treated with naproxen 250 $\mathrm{mg}$ orally twice daily, and hypothyroidism treated with thyroglobulin 1 grain orally daily. She also took Premarin therapy 21 days a month.

The patient reported a 20 year history of three to five watery stools a day which had been evaluated by several physicians. Previous investigations included multiple stool specimens negative for ova and parasites and bacterial pathogens, normal upper gastrointestinal and small bowel series, and normal barium enema. She had been treated unsuccessfully with Lomotil, Librax, loperamide, and methylcellulose. In April 1989 colonoscopy showed grossly normal mucosa, but colorectal biopsy specimens showed collagenous colitis (Figure (A)).

She was initially treated with sulphasalazine 1 $\mathrm{g}$ orally three times a day with no improvement of symptoms, and then prednisone $20 \mathrm{mg}$ orally twice a day was added. But she developed worsening non-bloody diarrhoea with 15 to 20 stools a day and crampy diffuse abdominal pain necessitating hospital admission. Colonoscopy done shortly after admission to hospital showed macroscopic mucosal erythema, granularity, friability, and exudate increasing in severity from rectum to transverse colon. A second set of mucosal biopsy specimens taken throughout the colon showed severe active chronic inflammation with severe crypt distortion, cryptitis, crypt abscesses, and ulceration consistent with ulcerative colitis (Figure (B)). She was treated with bowel rest, total parenteral hyperalimentation, prednisone $20 \mathrm{mg}$ orally three times a day, azathioprine $75 \mathrm{mg}$ orally four times a day, and sulphasalazine $1 \mathrm{~g}$ orally twice a day and naproxen was discontinued. On this regimen the diarrhoea slowly resolved over four weeks. Repeat flexible sigmoidoscopy in August 1989 showed mucosal granularity with loss of vascular pattern. Biopsy specimens from this procedure showed inactive ulcerative colitis with crypt distortion but no active inflammation or evidence of collagenous colitis (Figure (C)). Azathioprine was stopped and steroid tapered to $20 \mathrm{mg}$ per day. Presently the patient is passing three formed stools a day.

\section{Discussion}

Collagenous colitis is a newly recognised clinicopathological syndrome of unknown aetiology first reported by Lindstrom in $1976 .{ }^{1}$ Most patients are middle aged women who present with chronic watery diarrhoea. ${ }^{23}$ The colonic histopathology of this disorder is characterised by a band of collagen beneath the surface epithelium, increased plasma cells in the lamina propria, and in most cases increased intraepithelial lymphocytes. ${ }^{+}$

Discussion in published reports has focused on measuring the thickness of the subepithelial collagen band in diagnosing collagenous colitis. ${ }^{35}$ The thick collagen band is one facet of the histopathology, but other criteria are equally important in establishing this diagnosis. ${ }^{+67}$ As implied by the name, collagenous colitis is a mucosal inflammatory process. The inflammatory changes include expanded numbers of plasma cells in the lamina propria and, in most cases, damaged surface epithelium and increased intraepithelial lymphocytes. Moreover, subepithelial collagen can be recognised by qualitative as well as quantitative changes. Thus any increase in subepithelial collagenisation, in the proper inflammatory context, establishes the diagnosis of collagenous colitis. ${ }^{6}$

Recognising mucosal inflammation is essential, not only to correct interpretation of biopsy material but the inflammatory cells may also be 

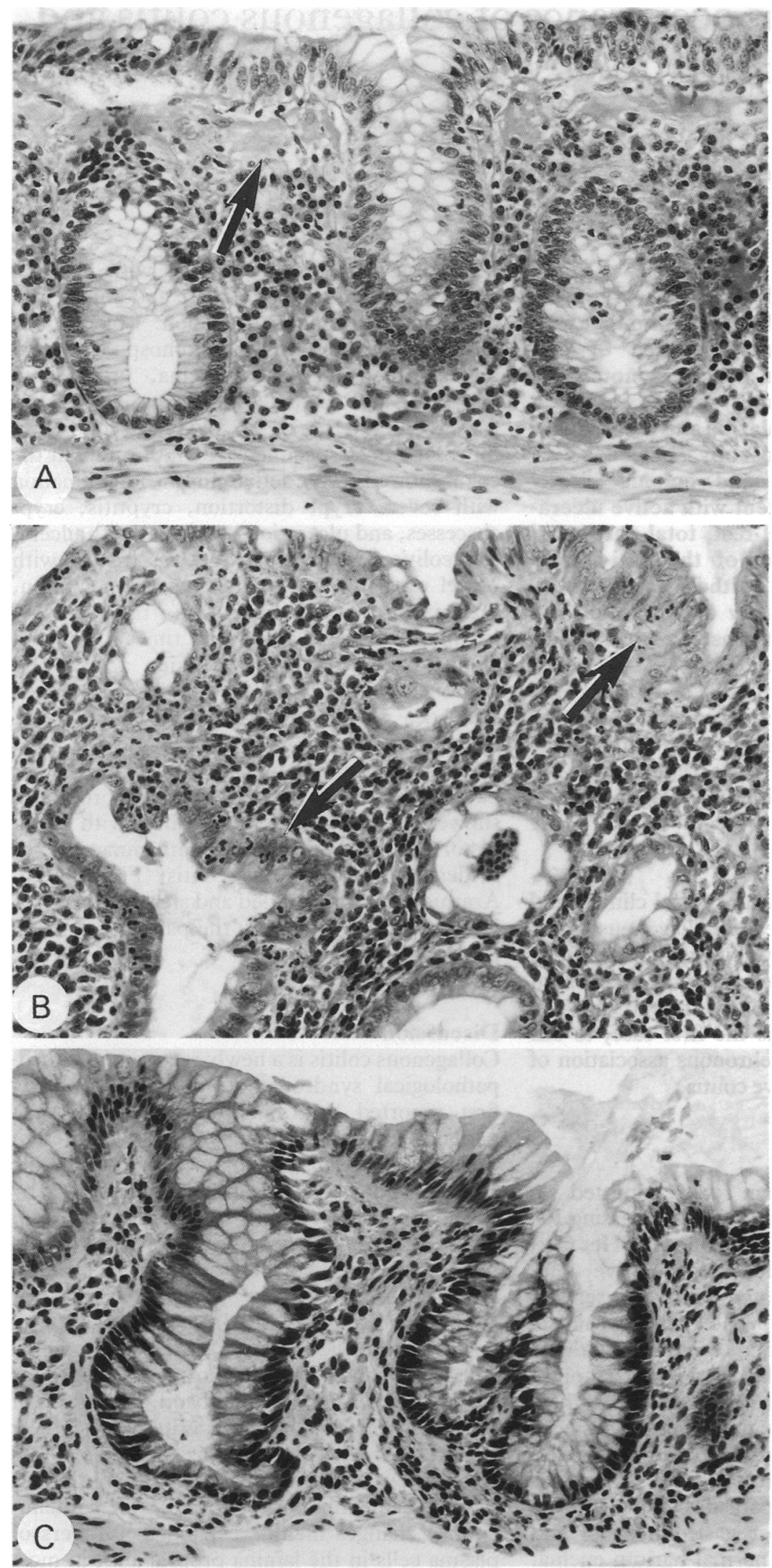

(A) The earliest set of biopsy specimens on this patient showed features typical of collagenous colitis. On this specimen and throughout the entire colorectum there was a mucosal inflammatory process characterised by increased plasma cells in the lamina propria, prominent intraepithelial lymphocytes, and a 20 micron thick subepithelial collagen band (arrow). (B) One month later biopsy specimens showed a very different histology with pronounced crypt distortion, a further increase in plasma cells, and neutrophilic infiltration of surface and crypt epithelium (arrows). This specimen was typical of the mucosa throughout the colorectum, and is consistent with ulcerative colitis. (C) One month later a subsequent specimen showed crypt distortion and fibrosis, but no continuing active inflammation and diminished chronic inflammation, consistent with inactive colitis. (All haematoxylin and eosin; original magnification $\times 240$.) important in the pathogenesis of collagenisation. Cytokines and other inflammatory mediators produced by inflammatory cells may provoke increased collagen synthesis from fibroblasts or myofibroblasts. ${ }^{8}$ As identified by immunohistochemical and ultrastructural studies, myofibroblasts are trapped in the subepithelial collagen layer in colorectal biopsy specimens from patients with collagenous colitis. ${ }^{9}$

This patient's initial clinical history and histopathology are typical for collagenous colitis. There was, however, an abrupt change in clinical symptomatology and histopathology, culminating in an illness consistent with ulcerative colitis. Though there has been one report of the simultaneous occurrence of Crohn's disease with collagenous colitis, ${ }^{10}$ this is the first report to our knowledge of the metachronous occurrence of ulcerative colitis in these patients.

Interestingly, this patient used a non-steroidal anti-inflammatory drug. In patients later diagnosed with collagenous colitis we have seen the exacerbation of diarrhoea with the use of these agents. " In addition, an appreciable proportion of patients with collagenous colitis take nonsteroidal anti-inflammatory drugs. Whether this represents use of an anti-inflammatory agent by a patient with secondary arthritis from collagenous colitis or a precipitating or exacerbating agent in this disease is unclear. As in this patient, however, withdrawing these drugs coincided with abatement of diarrhoea in other cases. ${ }^{11}$ Non-steroidal anti-inflammatory agents have been implicated in the exacerbation of ulcerative colitis. ${ }^{12}$

Collagenous colitis is essentially a newly recognised form of chronic colorectal mucosal inflammatory disease. ${ }^{4}$ As Lazenby et al have shown, the histopathological findings in collagenous colitis are distinct from other forms of inflammatory bowel diseases such as ulcerative colitis, Crohn's disease, acute 'self-limited' colitis, and lymphocytic colitis.' Though the cause of both ulcerative and collagenous colitis is unknown, different clinical and histological features favour a different cause of chronic mucosal inflammation. Moreover, we are unaware of the occurrence of both of these disorders in one patient in either the large cohort of inflammatory bowel disease patients followed up at our institution or in the 70 collagenous colitis patients followed up in our registry.

In this patient it is not known whether collagenous colitis represents an inflammatory insult which predisposes or initiates the development of ulcerative colitis or whether our patient exemplifies the chance occurrence of two different illnesses.

Supported in part by a research grant from the National Foundation of Ileitis and Colitis. AJL is the recipient of a fellowship from the National Foundation of Ileitis and Colitis.

We thank Drs John H Yardley, Theodore M Bayless, and Thomas R Hendrix for advice and support; and Linda $M$ Welch for secretarial help.

1 Lindstrom CG. 'Collagenous colitis' with watery diarrhoea new entity? Pathol Eur 1976; 11: 87-9.

2 Giardiello FM, Bayless TM, Jessurun J, Hamilton SR, Yardley JH. Collagenous colitis: physiologic and histopathologic studies in seven patients. Ann Intern Med 1987; pathologic

3 Rams H, Roberg AI, Ghandur-Mnaymneh L. Collagenous colitis. Ann Intern Med 1987; 106: 108-13. 
4 Jessurun J, Yardley JH, Giardiello FM, Hamilton SR, Bayless TM. Chronic colitis with thickening of the subepithelial collagen layer (collagenous colitis): histopathologic findings in 15 patients. Hum Pathol 1987; 18: 839-48.

5 Bogomeletz WV. Collagenous colitis: a clinicopathological review. Survey of Digestive Diseases 1983; 1 : 19-25.

6 Lazenby AJ, Yardley JH, Giardiello FM, Bayless TM. Pitfalls in the diagnosis of collagenous colitis. Experience with 75 cases from a registry of collagenous colitis at The Johns Hopkins Hospital. Hum Pathol (in press).

7 Lazenby AJ, Yardley JH, Giardiello FM, Jessurun J, Bayless T. Lymphocytic ('microscopic') colitis: a comparative histopathologic study with particular reference to collagenous colitis. Hum Pathol 1989; 20: 18-28.

8 Hwang WS, Kelly JK, Shaffer EA, Hershfield NB. Col- lagenous colitis: a disease of pericryptal fibroblast sheath? $\mathcal{F}$ Pathol 1986; 149: 33-40.

9 Widgren S, Jlidi R, Cox J. Collagenous colitis: histologic, morphometric, immunohistochemical and ultrastructura studies. Report of 21 cases. Virchows Arch A 1988; 413 287-96.

10 Chandratre S, Bramble MG, Cooke WM, Jones RA. Simultaneous occurrence of collagenous colitis and Crohn's disease. Digestion 1987; 36: 55-60.

11 Giardiello FM, Hansen FC, Lazenby AJ, et al. Collagenous colitis in the setting of nonsteroidal anti-inflammatory drugs and antibiotics. Dig Dis Sci 1990; 35: 257-60.

12 Kaufman HJ, Taubin HL. Nonsteroidal anti-inflammatory drugs activate quiescent inflammatory bowel disease. Ann Intern Med 1987; 107: 513-6. 